\title{
Phase Behavior and Induced Interdigitation in Bilayers Studied with Dissipative Particle Dynamics
}

\author{
Marieke Kranenburg, Maddalena Venturoli, and Berend Smit* \\ Department of Chemical Engineering, University of Amsterdam, Nieuwe Achtergracht 166, \\ 1018 WV Amsterdam, The Netherlands
}

Received: April 15, 2003; In Final Form: August 12, 2003

\begin{abstract}
Bilayers formed by coarse-grained models of amphiphilic surfactants are studied using dissipative particle dynamics combined with a Monte Carlo scheme to achieve the natural state of a tensionless bilayer. We address the issue of the influence of the molecular structure and the level of coarse graining on the bilayer properties by studying two models of different complexity: a single tail and a double tail surfactant. We compute the area per surfactant, the bilayer thickness, and the orientational order parameters and show how their dependence on the surfactant structure and temperature. We reproduce the gel to liquid crystalline phase transition, and we study the conditions that induce a gel interdigitated phase in the bilayer. We show how the interdigitated state in a bilayer containing double-tail surfactants can be reproduced by adding an extra bead in the surfactant headgroup, which mimics an ester-linkage to the phosphate group. In a bilayer formed by single tail surfactants, we induce interdigitation by changing the strength of the repulsive interaction between the headgroups, which corresponds with adding salt to the system. We are then able to derive a phase diagram as function of temperature and repulsion parameter for surfactants of different chain lengths.
\end{abstract}

\section{Introduction}

Knowledge of the phase behavior of phospholipids is important to understand the function of biological membranes. Many studies have been devoted to phosphatidylcholines (PCs). ${ }^{1}$ These lipids, comprising a phosphate containing headgroup and two hydrocarbon chains, form a stable bilayer at room temperature.

The phase behavior of different PCs has been determined experimentally (see ref 2 for a review). All PCs have a low temperature $L_{\beta}$ phase. In this phase, the bilayer is a gel: the chains of the phospholipids are ordered and show a tilt relative to the bilayer normal. At higher temperature, the $L_{\alpha}$ phase is the stable phase. This phase is the liquid crystalline state of the bilayer in which the chains are disordered, and tail overlap due to this thermal disorder is possible. This phase is physiologically the most relevant. ${ }^{1}$

Under normal conditions, a bilayer results from the joining of two phospholipid monolayers that contact each other at the terminal methyl group of their hydrophobic chains, whereas their hydrophilic headgroups are in contact with water. However, it is known experimentally that an interdigitated state, in which the terminal methyl groups of one monolayer interpenetrate the opposing layer, extending further than the bilayer midplane, is also possible. This state is present in bilayers in the gel phase. Interdigitation reduces the bilayer thickness, and this can, for example, affect the diffusion of ions across the bilayer or influence the activity of membrane proteins.

The interdigitated state in symmetric chain phospholipids, ${ }^{3}$ like dipalmitoylphosphatidylcholines (DPPC), can be induced by changes in the environment, like hydrostatic pressure or changes in the $\mathrm{pH}$ of the solution, ${ }^{4}$ or by incorporation, at the membrane interface, of small amphiphilic molecules, like

* To whom correspondence should be addressed. alcohols, ${ }^{5-7}$ or anesthetics. ${ }^{8}$ Interdigitation can also be induced by changes in the surfactant structure, for example by adding an ester-linkage in the headgroup of the phospholipids.

It has been proposed ${ }^{4,9}$ that specific interactions are not important in the formation of an interdigitated phase and that the main driving force that induces interdigitation is an increase in the headgroup surface area, which results in the creation of voids between the molecules. Because voids in the bilayer core are energetically unfavorable, they are filled up by molecules of the opposite monolayer.

This mechanism suggests that the formation of an interdigitated phase should be a general phenomenon independent of the specific chemical details of the lipid. This would imply that an interdigitated phase could also be induced in bilayers of, for example, single tail lipids. The fact that for single tail lipids the interdigitated phase has not been observed experimentally is one of the motivations to investigate the molecular aspects underlying the formation of an interdigitated phase in more detail.

Aggregates of amphiphilic molecules in solution, like membranes or micelles, can present a wide range of time and length scales. Theoretical studies on such systems have focused at different levels in the space and time resolution. Atomistic simulations represent explicitly all of the atoms in the molecules. However, the potentials necessary to reproduce the interactions between the different atoms are computationally demanding. Thus, these models permit an atomic resolution but on relatively short time scales, even if recently the progresses in computational techniques and the increased power of computers have allowed to reach time scales of several nanoseconds. ${ }^{10}$

In recent years, many efforts have been spent in developing coarse-grained models of amphiphilic surfactants to be able to describe phenomena which involve cooperative behavior, like self-assembly, bending modes, or phase transitions. The general characteristics of these models are to coarse grain some 
components of the system, by neglecting some of the atomic details, while preserving the essential aspects of the molecular structure. Examples of such mesoscopic models are systems in which the lipids are represented by chains of beads connected by a harmonic potential, each bead in the chain representing a group of atoms, rather than a single one. The main difference between mesoscopic models is the way in which the interactions between the particles are treated. The most used are of LennardJones type, ${ }^{1-13}$ or repulsive, soft potentials (dissipative particle dynamics DPD). ${ }^{14-16}$ In this work, we use DPD to simulate coarse-grained lipids. Dissipative particle dynamics ${ }^{17,18}$ is a technique which has been developed to simulate fluids at a mesoscopic level. Its main advantage is that, because of the soft interactions, much larger time and length scales are accessible, compared to an all-atom simulation.

An important question in the development of a mesoscopic model is how much chemical detail should be included in the model. To investigate the influence on the bilayer properties of the level of coarse graining, we compare two mesoscopic models of amphiphilic surfactants. The simplest model consists of a single hydrophilic head-bead connected to a hydrophobic tail. The second model we consider is a more realistic model of a phospholipid and consists of three hydrophilic head-beads and two hydrophobic tails. The presence of two tails is an example of a molecular detail that can be studied with our mesoscopic approach. One of the advantages of such mesoscopic models is that one does not have to assume a particular bilayer configuration, because the surfactants self-assembly into a stable bilayer, (inverted) micelles, or cylindrical micelles depending on the surfactant concentration. ${ }^{14}$ For both models, we study the temperature dependence of the area per surfactant and membrane thickness and show that we can qualitatively reproduce experimentally observed trends.

We briefly present the simulation techniques we used in section 2 , and in section 3 , we describe the model surfactants and define the properties used to characterize the bilayer structure. In section 4 , we will characterize the gel phase and the liquid crystalline phase and show that, both for a single tail and a double tail surfactant model, we can reproduce the transition between these two phases. We also observe that the two models can behave differently at low temperatures. We explain this difference in terms of the parameters of the model, with particular attention to the effective repulsion between the surfactant headgroups, by tuning the head-head repulsion parameter. For single tail surfactants, we will show that a high enough repulsion parameter between the headgroups is sufficient to induce interdigitation, whereas this is not the case for double tail surfactants. However, we are able to induce interdigitation in such surfactants by introducing an ester-linkage model in the headgroup.

\section{Simulation Methods}

2.1. Dissipative Particle Dynamics. A DPD bead represents the center of mass of a group of atoms clustered together. The beads interact via a force consisting of three contributions, all of them pairwise additive. The total force on particle $i$ can be written as the sum of the conservative, dissipation, and random forces

$$
f_{\mathrm{i}}=\sum_{i \neq j}\left(\boldsymbol{F}_{i j}^{\mathrm{C}}+\boldsymbol{F}_{i j}^{\mathrm{D}}+\boldsymbol{F}_{i j}^{\mathrm{R}}\right)
$$

The first term in the above equation represents a conservative force, which is usually soft repulsive of the form

$$
\boldsymbol{F}_{i j}^{\mathrm{C}}= \begin{cases}a_{\mathrm{ij}}\left(1-r_{\mathrm{ij}} / R_{\mathrm{c}}\right) \hat{\boldsymbol{r}}_{i j} & \left(r_{i j}<R_{\mathrm{c}}\right) \\ 0 & \left(r_{i j} \geq R_{\mathrm{c}}\right)\end{cases}
$$

where the coefficient $a_{i j}>0$ is a parameter expressing the maximum repulsion strength, $\boldsymbol{r}_{i j}=\boldsymbol{r}_{i}-\boldsymbol{r}_{j}$ is the distance between particles $i$ and $j$, and $R_{\mathrm{c}}$ is a cutoff radius which gives the extent of the interaction range.

The other two forces in eq 1 are a drag force $\left(F^{\mathrm{D}}\right)$ and a random force $\left(F^{\mathrm{R}}\right)$, of the form

$$
\begin{gathered}
\boldsymbol{F}_{i j}^{\mathrm{D}}=-\eta w^{\mathrm{D}}\left(r_{i j}\right)\left(\hat{\boldsymbol{r}}_{i j} \cdot v_{i j}\right) \hat{\boldsymbol{r}}_{i j} \\
\boldsymbol{F}_{i j}^{\mathrm{R}}=\sigma w^{\mathrm{R}}\left(r_{i j}\right) \zeta_{i j} \hat{\boldsymbol{r}}_{i j}
\end{gathered}
$$

where $v_{i j}=v_{i}-v_{j}$ is the velocity difference between particles $i$ and $j, \eta$ is the friction coefficient, and $\sigma$ is the noise amplitude. $\zeta_{i j}$ is a random number, independent for each pair of particles, taken from a uniform distribution. The combined effect of these two forces is a thermostat, which conserves (angular) momentum and, hence, gives the correct hydrodynamics at sufficient long time and length scales.

Español and Warren ${ }^{19}$ have shown that the equilibrium distribution of the system is the Gibbs-Boltzmann distribution if the weight functions and coefficients of the drag and the random force satisfy

$$
\begin{gathered}
w^{\mathrm{D}}(r)=\left[w^{\mathrm{R}}(r)\right]^{2} \\
\sigma^{2}=2 \eta k_{\mathrm{B}} T \\
w^{\mathrm{R}}(r)= \begin{cases}\left(1-r / R_{\mathrm{c}}\right) & \left(r<R_{\mathrm{c}}\right) \\
0 & \left(r \geq R_{\mathrm{c}}\right)\end{cases}
\end{gathered}
$$

If the weight function $w^{\mathrm{R}}(r)$ is chosen as in eq 6 , then, with eq 4 , all forces assume the same functional dependence on the interparticle distance $\boldsymbol{r}_{i j}$ as the conservative force $\boldsymbol{F}_{i j}^{\mathrm{C}}$.

The Newton's equations of motion are integrated using a modified version of the velocity Verlet algorithm. ${ }^{18}$ In DPD, it is convenient to use reduced units. ${ }^{15,18}$ The unit of length is defined by the cutoff radius $R_{\mathrm{c}}$, the unit of mass is defined by the masses $m$ of the particles (which are chosen to be the same for all the particles), and the unit of energy is defined by $k_{\mathrm{B}} T$. From these, the unit of time $\tau$ follows as

$$
\tau=R_{\mathrm{c}} \sqrt{m / k_{\mathrm{B}} T}
$$

2.2. Constant Surface Tension Simulations. In most molecular simulations of membranes, one uses a fixed number of surfactant molecules and a fixed area, combined with periodic boundary conditions. This corresponds to an infinitely large flat membrane. In general, such a system has a surface tension. It is an important question whether this corresponds to the surface tension of a real membrane. If not constrained, a membrane will adopt the conformation corresponding to the lowest free energy, i.e., a tensionless state. ${ }^{20}$ However, in an atomistic molecular dynamics study of a membrane, Feller and Pastor ${ }^{21,22}$ observed that a tensionless state did not reproduce the experimental value for the area per lipid. Their explanation of this result is that, because the typical fluctuations and out-of-plane variation of a macroscopic membrane do not develop in a small patch of a membrane, a positive surface tension (stretching) must be imposed in order to recover the experimental value of the area per lipid. Recently, Marrink and Mark ${ }^{10}$ simulated much larger patches of membranes up to 1800 lipids. Their calculations show that in a stressed membrane the area per lipid 
depends on the system size, or for a fixed area, the surface tension decreases if the system size is increased. This is in agreement with the conclusions of Feller and Pastor. On the other hand, it was found that, for a tensionless membrane, the equilibrium area does not depend on the system size and hence that there is no need to impose a surface tension to reproduce the experimental surface area in case of a stress free membrane.

Simulations at constant surface tension have been introduced by Chiu et al. in ref 23, and the constant surface tension ensemble $(N V T \gamma)$ has been considered in the literature. The corresponding equation of motion for molecular dynamics simulations have been derived by Zhang et al. in ref 24 . Here we use a different approach, based on a Monte Carlo (MC) scheme, to simulate a membrane at a given state of tension (of which the tensionless state is a particular case). We use an hybrid simulation scheme that combines DPD to evolve the positions of the particles and MC moves to change the shape of the simulation box. ${ }^{14}$

Consider a system with constant number of particles $N$, constant temperature $T$, and constant volume $V$, in which an interface of area $A$ is present. The work done on the system by compression or stretching of the interface by $\mathrm{d} A$, is given by ${ }^{25}$ $\mathrm{d} W=\gamma \mathrm{d} A$, where $\gamma$ is the surface tension. The partition function for such a system can then be written as

$$
Q=\frac{1}{\Lambda^{3 \mathrm{~N}} N !} \int_{V} \mathrm{~d} \boldsymbol{r}^{\mathrm{N}} \exp \left[-\beta\left(U\left(\boldsymbol{r}^{\mathrm{N}}\right)-\gamma A\right)\right]
$$

If we take a rectangular simulation box, with dimensions $L_{\|}$ parallel to the interface ( $x y$ plane), and $L_{\perp}$ perpendicular to the interface ( $z$ axis), so that the system volume is $V=L_{\perp} L_{\|}^{2}$ and the area of the interface $A=L_{\|}^{2}$, we define a transformation of the box size which changes the area and the height but keeps the volume constant. Such a transformation can be written in the form

$$
\begin{aligned}
& L_{\|}^{\prime}=\lambda L_{\|} \\
& L_{\perp}^{\prime}=\frac{1}{\lambda^{2}} L_{\perp}
\end{aligned}
$$

where $\lambda$ is the parameter of the transformation.

By changing $\lambda$, the above expression generates a transformation of coordinates which preserves the total volume of the system; hence, no work against the external pressure is performed. The coordinate phase space has now an extra degree of freedom, represented by the parameter $\lambda$. To write the partition function corresponding to this ensemble, it is convenient to first introduce a set of scaled coordinates $s \in[0,1]$, defined as

$$
\boldsymbol{r}=\left(L_{||} s_{x}, L_{||} s_{y}, L_{\perp} s_{z}\right)
$$

By a transformation of the box dimensions with $\lambda$, the coordinates of the particles rescale as

$$
\boldsymbol{r}^{\prime}=\left(\lambda L_{||} s_{x}, \lambda L_{||} s_{y}, \frac{1}{\lambda^{2}} L_{\perp} s_{z}\right)
$$

In terms of these scaled coordinates, the partition function of the system is

$$
Q=\frac{V^{\mathrm{N}}}{\Lambda^{3 N} N !} \int \mathrm{d} \lambda \int \mathrm{d} \boldsymbol{s}^{N} \exp \left\{-\beta\left[U\left(\boldsymbol{s}^{\mathrm{N}} ; \lambda\right)-\gamma A(\lambda)\right]\right\}
$$

The probability of finding a configuration with scaled positions

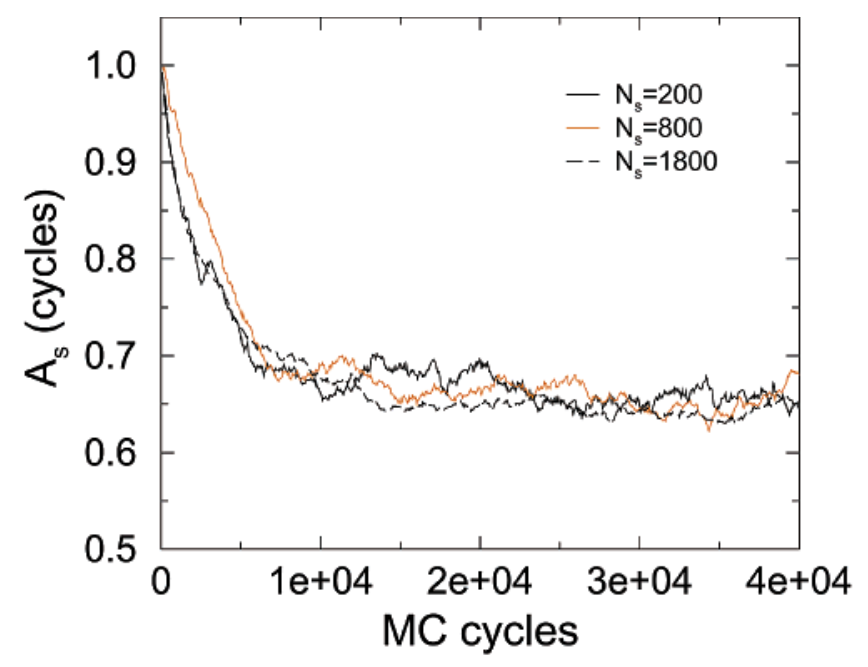

Figure 1. Instantaneous area per surfactant $A_{\mathrm{s}}$, as function of the number of MC cycles, for bilayer patches of different sizes. The lines correspond to bilayers of 200,800 , and 1800 surfactants.

$\boldsymbol{s}^{N}$ and parameter $\lambda$ is then given by

$$
N\left(s^{N}, \lambda\right) \propto \exp \left\{-\beta\left[U\left(s^{N} ; \lambda\right)-\gamma A(\lambda)\right]\right\}
$$

In a MC move, an attempt of changing the parameter $\lambda \rightarrow \lambda^{\prime}$ is then accepted with a probability

$$
P_{\text {acc }}\left(\lambda \rightarrow \lambda^{\prime}\right)=\frac{\exp \left\{-\beta\left[\left(U\left(s^{\prime N} ; \lambda^{\prime}\right)-\gamma A\left(\lambda^{\prime}\right)\right]\right\}\right.}{\exp \left\{-\beta\left[\left(U\left(s^{N} ; \lambda\right)-\gamma A(\lambda)\right]\right\}\right.}
$$

The described scheme can be applied to impose any value of the surface tension, in our simulations to obtain a tensionless state $(\gamma=0)$. It is important to remark that this scheme assumes that the stress tensor is diagonal. This is not true for solid systems. We will show that, even when we have a bilayer in the gel phase, the system is still fluid enough for the method to be applied.

To test finite size effects, we performed a series of simulations of bilayers of different sizes (200, 800, and 1800 surfactants), to which we imposed the condition of zero surface tension using the described hybrid MC-DPD scheme. In Figure 1, the area per molecule as a function of the number of MC cycles is plotted for these different systems. After a period of equilibration, all of the bilayers converge to the same value of the area per molecule. Our results are in agreement with the ones of Marrink and Mark; that is, the area per surfactant in a tensionless bilayer does not depend on the system size.

2.3. Model Surfactants. We consider three different types of particles: water-like particles (w), hydrophilic particles (h), which represent beads in the headgroup of the surfactants, and hydrophobic particles ( $\mathrm{t}$ ), which represent beads in the tail of the surfactants.

The value of the repulsion parameter for water-like particles is chosen such that the simulated compressibility of DPD water at room temperature corresponds to the experimental value. ${ }^{18,26}$ Groot and co-workers assume that one water bead represents one water molecule. If the bead density of the system is chosen equal to $\rho R_{\mathrm{c}}{ }^{3}=3$, the correct compressibility of water is obtained for $a_{\mathrm{ww}}=25 k_{\mathrm{B}} T$. To model the amphiphilic nature of the surfactants, the repulsion parameters $a_{i j}$ (eq 2) between two beads which are both hydrophilic (hydrophobic) are smaller than the ones between hydrophilic and hydrophobic beads. The parameters describing these interactions are derived using a correspondence with the Flory-Huggins model for mutual 
TABLE 1: Repulsion Parameters $a_{i j}$ (eq 2)

\begin{tabular}{llll}
\hline & $(\mathbf{w})$ & $(\mathbf{h})$ & $(\mathbf{t})$ \\
\hline$(\mathbf{w})$ & 25 & 15 & 80 \\
$(\mathbf{h})$ & 15 & 35 & 80 \\
$(\mathbf{t})$ & 80 & 80 & 25
\end{tabular}

solubilities. ${ }^{18,26}$ It is important to stress that, with a different choice of the level of coarse-graining, the repulsion parameters will have different values. ${ }^{15}$ The interaction parameters we used in this work (shown in Table 1) are the same as those used by Groot in ref 26, with the exception of $a_{\mathrm{tt}}$ (tail-tail), which we increased from 15 to 25 to avoid unrealistic high densities in the bilayer hydrophobic core.

From this coarse-graining procedure, the interaction parameters are defined in units of $k_{\mathrm{B}} T$. To use reduced units, we define $k_{\mathrm{B}} T_{\mathrm{o}}=1$ where $T_{\mathrm{o}}$ is room temperature. The interaction parameters can then be expressed in these reduced units; that is, the $a_{\mathrm{ww}}$ parameter has been fitted to give the correct compressibility of water at room temperature and at the assumed density. In principle, we could use the same procedure to match the compressibility of water at different temperatures. This gives, however, a temperature dependent $a$ parameter which would make the interpretation of our results more complex. Therefore, we have chosen to keep the parameters fixed and only change the temperature. In the following, we will use the notation $T^{*}$ to indicate the reduced temperature.

The surfactants are built connecting the beads by springs in the form

$$
\boldsymbol{F}_{\text {spring }}=-K_{\mathrm{r}}\left(r_{i j}-r_{\mathrm{eq}}\right) \hat{\boldsymbol{r}}_{i j}
$$

The equilibrium distance is set at $r_{\mathrm{eq}}=0.7$. We determined the spring constant $K_{\mathrm{r}}$ by demanding that in a typical surfactant $98 \%$ of the bond distance distribution lies within one $R_{\mathrm{c}}$. The value $K_{\mathrm{r}}=100$ was found to satisfy this requirement.

We studied two surfactants types (see Figure 2). The simplest one consists of one head bead connected to a single tail. If we compare this surfactant with real phospholipids, noticeable differences, at a coarse grain level, are the size of the headgroup and the double tail in the real phospholipids. To study the effect of these differences, we also simulate a model surfactant with three head beads and two tails. In both models, the tail(s) can have different lengths. We denote a single-tail surfactant by $h t_{\mathrm{N}}$ and a double-tail surfactant by $h_{3}\left(t_{\mathrm{N}}\right)_{2}$, where in both cases $N$ is the number of beads in the tail(s).

To control the chain flexibility, an extra bond-bending force between consecutive bonds is added in the form

$$
\begin{gathered}
\boldsymbol{F}_{\theta}=-\nabla U_{\theta} \\
U_{\theta}=\frac{1}{2} K_{\theta}\left(\theta-\theta_{0}\right)^{2}
\end{gathered}
$$

where $K_{\theta}$ is the bending constant, $\theta$ is the angle between two consecutive bonds, and $\theta_{0}$ is the equilibrium angle. To find the values of $K_{\theta}$ and $\theta_{0}$, we performed a molecular dynamics simulation of a phospholipid in water yielding a bending constant of $K_{\theta}=10$ and an equilibrium angle of $\theta_{0}=\pi$ for all bonds in the tail. This value of $\theta_{0}$ corresponds to saturated hydrocarbon chains. In the double-tail surfactant, a bond bending potential is also applied between the vectors connecting the tails to the headgroup, with $\theta_{0}=(1 / 2) \pi$ and $K_{\theta}=3$. The head group of the double tail surfactants is fully flexible.

2.4. Computational Details. All our simulations are performed on a tensionless bilayer of 200 surfactants. The number

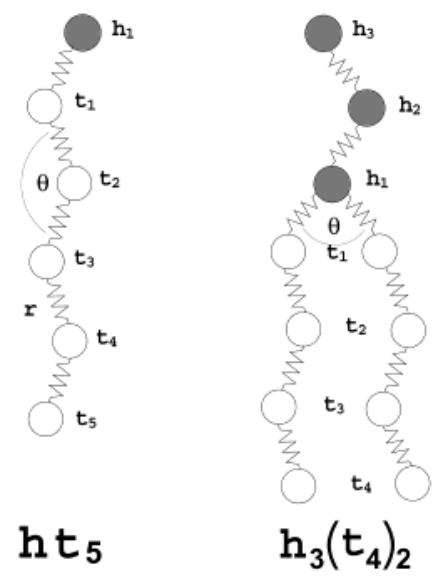

Figure 2. Two model surfactants used in this study: single tail surfactant (left) and double tail surfactant (right). The black particles represent the head beads and the white particles the tail beads.

of water particles is chosen such that a bilayer does not have any interaction with its periodic image. This results in 15002100 water particles for the single-tail surfactants and 45007000 water particles for double-tail surfactants. The overall density of the system is $\rho=3$. We initialize our system by distributing lipids randomly in water, and we observe the selfassembly of a bilayer using DPD simulation only. The equations of motions are integrated with $\Delta t=0.03$. After the bilayer is formed, we perform, in addition to the DPD moves, Monte Carlo moves in which we change the area as well. A typical simulation required 100000 cycles of which 20000 cycles were needed for equilibration. Per cycle it is randomly chosen if 50 DPD time steps are performed or an attempt to change the area of the box was made. We have optimized the relative number of $\mathrm{MD}$ and MC moves to sample as efficiently as possible. On average in $70 \%$ of the cycles, DPD time steps are performed. The volume of the system remained constant during all simulations. This implies that as the temperature is changed the total pressure also (slightly) changes; the pressure ranges from 20.9 to 21.8 in the temperature range applied.

2.5. Bilayer Properties. In this section we define the quantities used to characterize the bilayer phases.

2.5.1. Area per Molecule and Bilayer Thickness. An important quantity measured in experiments on lipid membranes is the area per molecule. To compute this quantity, we simply divide the total projected area in the $x y$ plane (after equilibration and at a state of zero surface tension) by half the number of surfactants in the bilayer, because, on average, the number of molecules in each side of the bilayer is equal.

Another quantity used to characterize the bilayer structure is the thickness of the membrane, $D_{\mathrm{hh}}$, defined as the average distance between the headgroups of opposing surfactants. From the density profiles obtained in our simulations, we compute $D_{\text {hh }}$ as the distance between the headgroup density peaks in the two monolayers. For double tail surfactants, which have three head beads, the reference bead to compute $D_{\mathrm{hh}}$ is the bead connecting the two tails (bead $h_{1}$ in Figure 2).

2.5.2. Chain Overlap and Interdigitation. To further characterize the bilayer structure and to investigate the presence of an interdigitated phase, we characterize the extent of interpenetration of the hydrophobic tails of the surfactants on opposite sides of the bilayer, by defining the chain overlap $D_{\text {overlap, as }}$

$$
D_{\text {overlap }}=\frac{2 L_{z}-D_{\mathrm{c}}}{L_{z}}
$$


$L_{z}$ is the average chain length in the direction normal to the bilayer, i.e., the average distance projected on the bilayer normal ( $z$ axis) between the first bead in the tail $t_{1}$ and the last one $t_{\mathrm{N}}$

$$
L_{\mathrm{z}}=\left|\left\langle z_{\mathrm{t} 1}\right\rangle-\left\langle z_{\mathrm{tN}}\right\rangle\right|
$$

$D_{\mathrm{c}}$ is the thickness of the hydrophobic core, that we compute as the average distance along the bilayer normal between the first bead (or beads in case of double tail surfactants) in the tail(s) of the surfactants in one layer and the ones in the opposite layer

$$
D_{\mathrm{c}}=\left|\left\langle z_{\mathrm{t} 1}^{1}\right\rangle-\left\langle z_{\mathrm{t} 1}^{2}\right\rangle\right|
$$

where $\left\langle z_{\mathrm{t} 1}^{i}\right\rangle$ is the average $z$ position of the first tail bead(s) of the surfactants in monolayer $i$.

We will see in the next sections that at high temperatures the chain overlap is mainly due to the disorder of the molecules, whereas at low temperatures, the chain overlap can be seen as an effective interdigitation of the chains.

2.5.3. Orientational Order Parameters. Another quantity used in determining the structure of lipid bilayers is the orientational order parameter. The order parameter can be directly measured by deuterium substitution NMR spectroscopy and is given by

$$
S=\frac{1}{2}\left\langle 3 \cos ^{2} \theta-1\right\rangle
$$

where $\theta$ is the angle between the orientation of the vector along a given $\mathrm{C}-\mathrm{H}$ bond and the bilayer normal.

In our mesoscopic model, however, the hydrogen atoms are not present; hence, we must use a different definition. The mathematical expression is the same as in eq 21 , but the angle $\theta$ is now defined as the angle between the orientation of the vector along two beads in the chain and the bilayer normal

$$
\cos \theta=\frac{\boldsymbol{r}_{i j} \cdot \hat{\boldsymbol{n}}}{r_{i j}}=\frac{z_{i j}}{r_{i j}}
$$

where $\hat{\boldsymbol{n}}$ is a unit vector normal to the bilayer and $\boldsymbol{r}_{i j}=\boldsymbol{r}_{i}-\boldsymbol{r}_{j}$ is the vector between beads $i$ and $j\left(r_{i j}=\left|\boldsymbol{r}_{i j}\right|\right)$. The order parameter has value 1 if this vector is on average parallel to the bilayer normal, 0 if the orientation is random, and -0.5 if the bond is on average parallel to the bilayer plane.

With this definition of the angle $\theta$, we can compute the order parameter for a vector between any two beads in the surfactant. In particular, we are interested in characterizing the overall order of the chains and the local order. For the first quantity, we define the indexes of the vector $\boldsymbol{r}_{i j}$ in eq 22 as $i=t_{\mathrm{N}}$ and $j=t_{1}$, where $t_{\mathrm{N}}$ is the last bead in the surfactant tail and $t_{1}$ is the first one (see Figure 2). We call $S_{\text {chain }}$ the corresponding order parameter. For the local order, we define $i=t_{n+1}$ and $j=t_{n}$ with the index $n$ increasing going toward the tail end and call the corresponding order parameter $S_{n}$. If $n$ is taken progressively from the headgroup to the tail-end of the molecule, a plot of the corresponding order parameters, $S_{n}$, gives an indication of the persistence of order through the bilayer core.

\section{Results}

3.1. Single-Tail Surfactants. In this section, we present results of simulations of a bilayer formed by single tail surfactants consisting of one head bead and nine tail beads $\left(h t_{9}\right)$, which we study at different reduced temperatures, from $T^{*}=$ 0.8 to $T^{*}=1.5$.

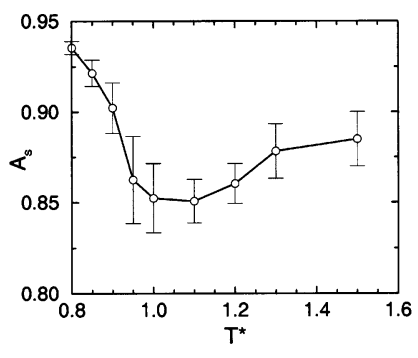

(a)

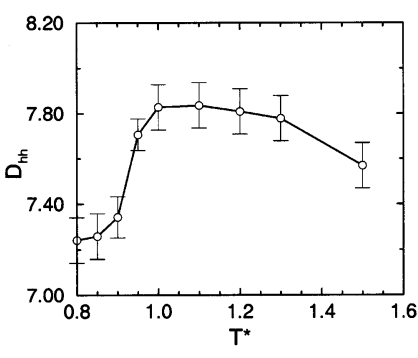

(b)
Figure 3. Area per surfactant $A_{\mathrm{s}}$ and (b) bilayer thickness $D_{\mathrm{hh}}$ as function of reduced temperature $T^{*}$ for surfactant type $h t_{9}$.

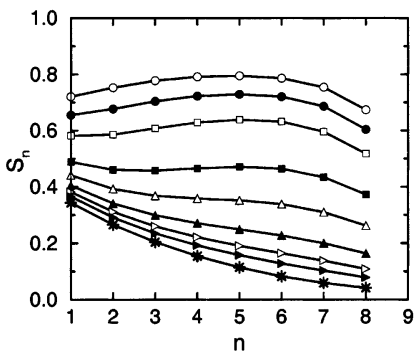

(a)

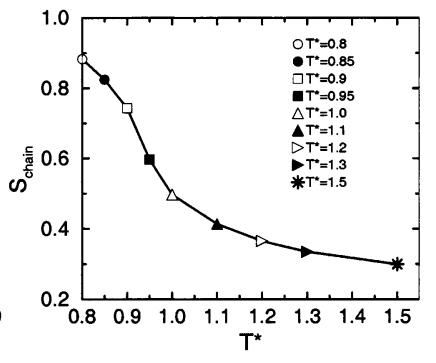

(b)
Figure 4. (a) Local order parameter $S_{n}$ and (b) chain order parameter $S_{\text {chain }}$, as function of reduced temperature $T^{*}$.

3.1.1. Bilayer Structure. In Figure 3, the average area per surfactant $A_{\mathrm{s}}$ and the bilayer thickness, $D_{\mathrm{hh}}$, are plotted as function of temperature. The error bars have been calculated with the block averages method. ${ }^{27,28}$ In all the other plots, we will show of the area per surfactant or bilayer thickness, and we will not include error bars, which, however, have been estimated as $\leq 5 \%$.

In Figure 3, we can distinguish two regions: at low temperatures, the area per surfactant is decreasing with increasing temperature and the thickness is increasing, whereas at high temperatures the area is increasing with increasing temperature, and the thickness is decreasing. At the lowest temperature studied $\left(T^{*}=0.8\right)$, the area is larger than the area at the highest temperature studied $\left(T^{*}=1.5\right)$, whereas the thickness at $T^{*}=$ 0.8 is smaller than the thickness at $T^{*}=1.5$. This different temperature dependence of $A_{\mathrm{s}}$ and $D_{\mathrm{hh}}$ suggests that the bilayer undergoes a phase transition. Before discussing this transition in detail we will first characterize the low and the hightemperature phases.

To characterize the ordering of the surfactants in the bilayer, we use the order parameters $S_{\text {chain }}$ and $S_{n}$. In Figure 4, the values of both $S_{\text {chain }}$ and $S_{n}$ are plotted as function of temperature. The high values of $S_{n}$ at temperatures below $T^{*}=0.95$ indicate that the bonds are ordered along the bilayer normal. This order persists even for bonds far from the headgroup region, decreasing slightly with increasing temperature. Above $T^{*}=0.95$, the values of $S_{n}$ further decrease with increasing temperature, and the order along the chain is lost.

The overall order of the chains $\left(S_{\text {chain }}\right)$ shows a similar behavior. Also here we can distinguish two regions: below $T^{*}$ $=0.95$ where $S_{\text {chain }}$ has values higher than 0.5 indicating that the chains are ordered along the bilayer normal, and above $T^{*}$ $=0.95$ where the values of $S_{\text {chain }}$ decrease below 0.5 , showing an increase in the disorder of the chains.

To further characterize the structure of the bilayer in the low and high-temperature regions, in Figure 5, we compare the inplane radial distribution function $g(r)$ of the head beads of the surfactants at one interface, for two different temperatures: $T^{*}$ 


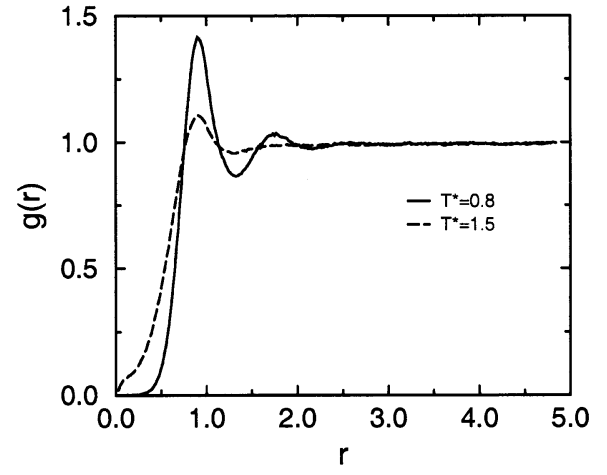

Figure 5. Two-dimensional radial distribution function $g(r)$ in the bilayer plane for the headgroups at $T^{*}=0.8$ and 1.5.

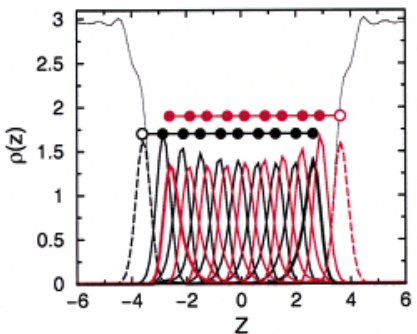

(a) $\mathrm{T}^{*}=0.8$

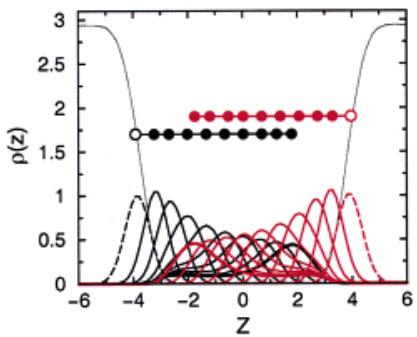

(c) $\mathrm{T}^{*}=1.0$

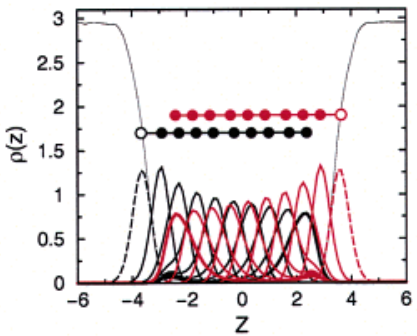

(b) $\mathrm{T}^{*}=0.9$

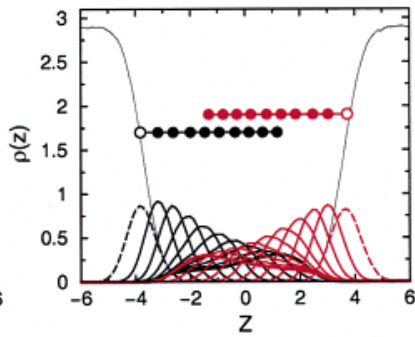

(d) $\mathrm{T}^{*}=1.5$
Figure 6. Density profiles $\rho(z)$ along the bilayer normal $z$ for different reduced temperatures $T^{*}$. Each line is the density profile for a different bead: full lines are the densities of the tail beads, dashed lines are the densities of the head beads, and the thin solid line is the density of water. The black lines correspond to the surfactants in one monolayer, whereas the red lines correspond to the surfactants in the opposite monolayer. The big dots correspond to the maxima in the bead density distributions and illustrate the position of the beads in the bilayer. The full circles correspond to tail beads and the open circles to head beads.

$=0.8$ and $T^{*}=1.5$. It is important to note that, at the lowest temperatures studied, the system is not solid. This allows one to apply the constant surface tension scheme. At $T^{*}=0.8$, the radial distribution function shows more pronounced peaks compared to the $g(r)$ at $T^{*}=1.5$, which corresponds to a more structured organization of the surfactants headgroups in the bilayer plane. The structure in the radial distribution function and the high values of the order parameters for low temperatures suggest that the low-temperature phase is the ordered gel phase, whereas at high temperatures, the bilayer is in the disordered liquid crystalline phase.

In Figure 6, we show the density profiles in the direction normal to the bilayer for the system components at different reduced temperatures. Figure 6, parts a and b, corresponds to a bilayer in the gel phase, whereas parts $\mathrm{c}$ and $\mathrm{d}$ correspond to a bilayer in the liquid crystalline phase.

It is clearly visible that, in the low-temperature region, the two monolayers are interdigitated. At $T^{*}=0.8$, the overlap extends up to the eighth bead in the tail, and the peaks of the

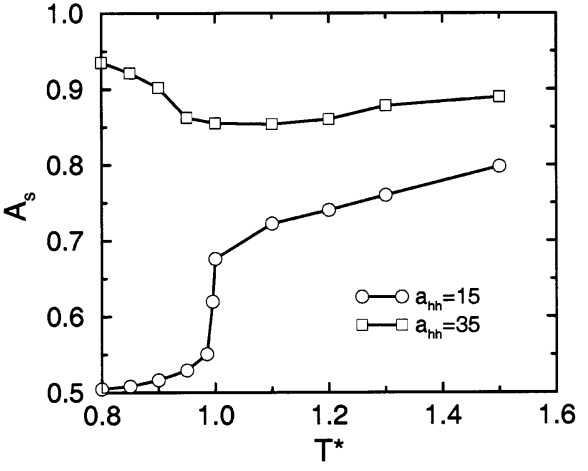

Figure 7. Comparison of the area per surfactant $A_{\mathrm{s}}$, as function of reduced temperature $T^{*}$, for two different repulsion parameters between the surfactant headgroups: $a_{\mathrm{hh}}=15$ (circles) and $a_{\mathrm{hh}}=35$ (squares).

density profiles for the surfactants tail beads in one monolayer (black full lines) are exactly alternating with the peaks of the opposite monolayer (red full lines), showing an optimal packing of the tails. This structure resembles the experimentally observed interdigitated phase $L_{\beta \mathrm{I}}$.

We can now explain the temperature dependence of the area per surfactant (Figure 3). The low-temperature phase is the interdigitated gel $L_{\beta \mathrm{I}}$. In this phase, the ordering of the chains is the dominating effect. The surfactants stretch out in the direction normal to the bilayer, inducing interdigitation. This packing results in a larger average distance between the surfactants headgroups in each monolayer and in a larger area. In this region, an increase of temperature reduces the values of the order parameter (Figure 4b), but along the chain the order persists (Figure 4a). Thus, interdigitation is still present but is decreasing in depth, resulting in an increase of the bilayer thickness and a decrease of the area per surfactant. Above the transition temperature, the chains loose the persisting order and are not interdigitated. Only the terminal tail beads overlap, because of thermal disorder. In this temperature region, an increase in temperature increases the effective volume occupied by the molecules, but the extent of chain overlap does not depend significantly of temperature. As a result, the area per molecule increases while the bilayer thickness decreases.

3.1.2. Interdigitated and Bilayer Phases. In the previous section, we have seen that single tail surfactants form spontaneously an interdigitated phase at low temperatures, whereas the most common organization of (symmetric) phospholipids in membranes is a bilayer formed by two separate monolayers. ${ }^{29}$ It is therefore interesting to investigate whether we can adapt the single tail model to reproduce the phase behavior of real membranes, and in particular if we are able to obtain a noninterdigitated gel phase. If the main cause of interdigitation is an increase in the headgroups surface area, ${ }^{4,9}$ we can test this mechanism by changing the value the headgroup repulsion parameter, $a_{\mathrm{hh}}$, in our model. Taking as initial condition the interdigitated bilayer at $T^{*}=0.85$, we decreased the headgroup repulsion parameter from $a_{\mathrm{hh}}=35$ to $a_{\mathrm{hh}}=15$, the latter being the same repulsion parameter as between an hydrophilic bead and a water-bead. Experimentally, changing the head-head interactions corresponds to, for example, adding salt to the system. It is important to recall that, with the zero surface tension scheme, the system can evolve to the optimum area per surfactant even if the bilayer undergoes structural rearrangements.

Figure 7 shows the temperature dependence of the area per surfactant for the repulsion parameters $a_{\mathrm{hh}}=35$ and $a_{\mathrm{hh}}=15$. We observe that the behavior in temperature of the area per 


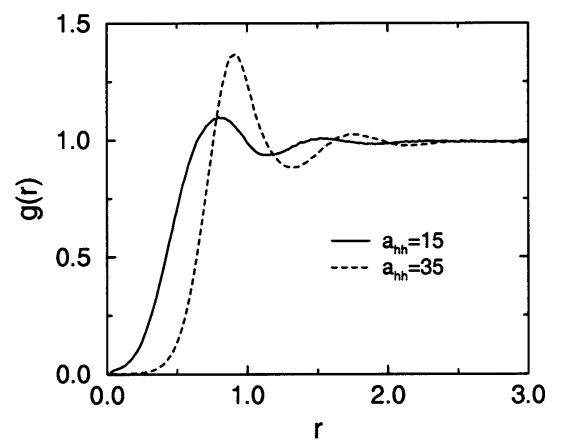

Figure 8. Two-dimensional radial distribution function $g(r)$ in the bilayer plane for the headgroups at $T^{*}=0.85$, for $a_{\mathrm{hh}}=15$ (solid line) and $a_{\mathrm{hh}}=35$ (dashed line).

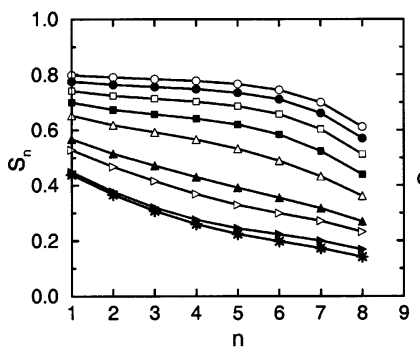

(a)

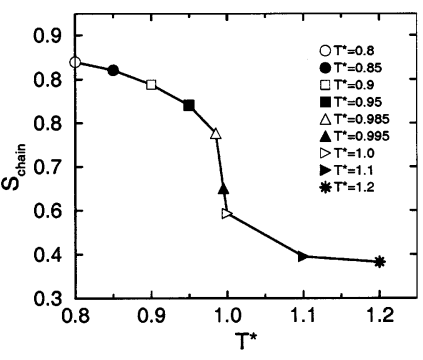

(b)
Figure 9. (a) Local order parameter $S_{n}$ and (b) chain order parameter

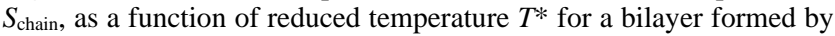
surfactants with $a_{\mathrm{hh}}=15$.

surfactant for the two values of $a_{\mathrm{hh}}$ is very different. At low temperatures, the area at $a_{\mathrm{hh}}=35$ is almost twice the value of the area at $a_{\mathrm{hh}}=15$. The decrease of the headgroup surface area is also shown in Figure 8, where we compare the radial distribution functions of the headgroups in the bilayer plane at $T^{*}=0.85$. The peaks in the radial distribution function for the system with $a_{\mathrm{hh}}=15$ (solid line) are shifted to the left compared to the system with $a_{\mathrm{hh}}=35$ (dashed line), showing a decrease of the distance between the headgroups. This is a strong indication that at low temperature, with the lower repulsion parameter, the bilayer is in the $L_{\beta}$ phase.

To further characterize the bilayer structure for $a_{\mathrm{hh}}=15$, we study the order parameters $S_{\text {chain }}$ and $S_{n}$, which are plotted in Figure 9 . At temperatures $T^{*} \leq 0.95$, the chains are locally ordered (values of $S_{n}$ above 0.5 ), and the order does not decrease significantly going through the hydrophobic core. Also the overall order of the chains $S_{\text {chain }}$ is high in this temperature region. Above $T^{*}=0.95$, we observe a decrease in both the order parameters. The chains become disordered, and the persistence of order along the chain is lost. This trend is analogous to the one observed for $a_{\mathrm{hh}}=35$. In both cases, the low-temperature region is characterized by the ordering of the chains, whereas at high temperatures, the chains are disordered. However, although for $a_{\mathrm{hh}}=35$ the two monolayers are interdigitated in the ordered phase, for $a_{\mathrm{hh}}=15$ the ordered phase is a bilayer formed by two separated leaflets. This can clearly be seen from the density profiles, which we plot as function of reduced temperature in Figure 10.

This figure shows that the melting of the bilayer results in a broader shape of the density profiles. The increase of disorder in the chains (see Figure 9) results in a partial overlap of the two monolayers. This transition upon heating is also reflected in the trend of the area per surfactant with temperature (Figure 7), which shows a sharp increase between $T^{*}=0.95$ and $T^{*}=$ 1.0. We can then conclude that a transition from an ordered to

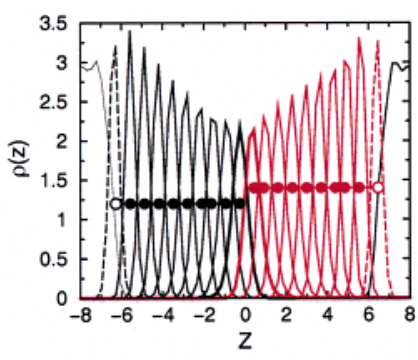

(a) $\mathrm{T}^{*}=0.8$

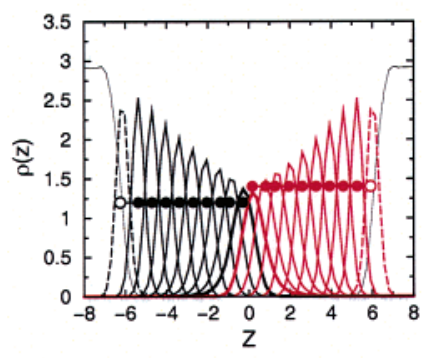

(c) $\mathrm{T}^{*}=0.95$

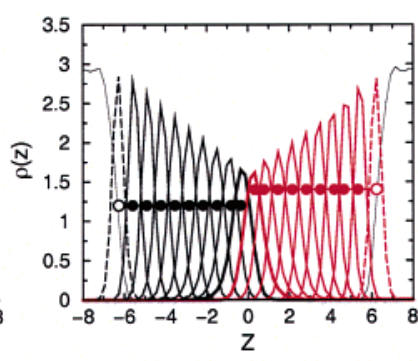

(b) $\mathrm{T}^{*}=0.9$

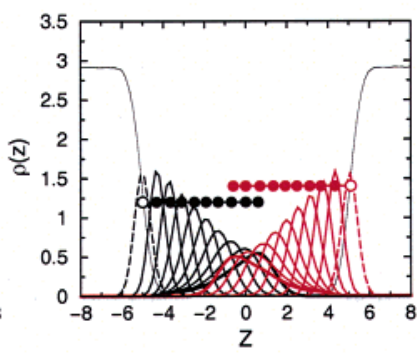

(d) $\mathrm{T}^{*}=1.0$
Figure 10. Density profiles as function of temperature for a bilayer formed by surfactants with $a_{\mathrm{hh}}=15$ (see also the caption to Figure 6).

a disordered phase takes place at a temperature $0.95<T_{\mathrm{m}}<$ 1.0 .

We have thus shown that the bilayer structure in the lowtemperature region depends on the repulsion between the surfactant headgroups. By tuning this parameter, we can obtain both the gel phase $L_{\beta}$ and the interdigitated gel phase $L_{\beta \mathbf{I}}$. Experimentally, both in the liquid crystalline phase ${ }^{30}$ and in the gel phase, ${ }^{31}$ a monotonic increase of the area per lipid is observed when the temperature is increased. This is caused by an increase in the disorder of the tails. ${ }^{30}$ For the low repulsion parameter of $a_{\mathrm{hh}}=15$, we reproduce the experimental observed trends. It is worth mentioning that, in most cases, in the gel phase the phospholipid chains are tilted respect to the bilayer normal ${ }^{2}$. Although with single tail surfactants we do not observe any tilt, we will see in the next sections that the double tail surfactants are tilted in the gel phase ( $L_{\beta^{\prime}}$ phase).

3.1.3. Phase Behavior as Function of Head-Head Repulsion and Tail Length. It is now interesting to do a more systematic study of these phase transitions for a range of repulsion parameters. The phase transitions we consider are: (1) transition from interdigitated gel to gel $\left(L_{\beta \mathrm{I}} \rightarrow L_{\beta}\right)$; (2) transition from interdigitated gel to liquid crystalline $\left(L_{\beta \mathrm{I}} \rightarrow L_{\alpha}\right)$; (3) transition from gel to liquid crystalline $\left(L_{\beta} \rightarrow L_{\alpha}\right)$. As we have shown, the first transition is induced by a decrease in the repulsion parameter $a_{\mathrm{hh}}$, whereas the latter ones are temperature dependent.

We use three quantities to distinguish among the different phases: the area per surfactant $A_{\mathrm{s}}$, the extent of tail overlap

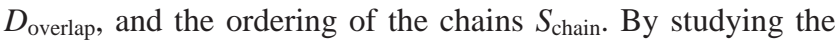
behavior of these quantities as function of temperature and head-head repulsion parameter, we can determine the phase diagram of $h t_{9}$ as shown in Figure 13d.

In Figure 11, we plot the area per surfactant $A_{\mathrm{s}}$ and the extent of tail overlap $D_{\text {overlap }}$ as function of temperature and headhead repulsion parameter. For repulsion parameters $a_{\mathrm{hh}} \leq 18$, the low-temperature phase is the bilayer gel $L_{\beta}$ phase, whereas for repulsion parameters $a_{\mathrm{hh}}>18$, the low-temperature phase is the interdigitated gel $L_{\beta \mathrm{I}}$.

By increasing temperature, all of the bilayers melt from an ordered into a disordered phase. For bilayers in the $L_{\beta}$ phase, the area per molecule and chain overlap increase upon melting, 


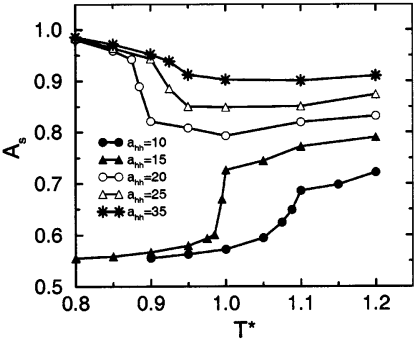

(a)

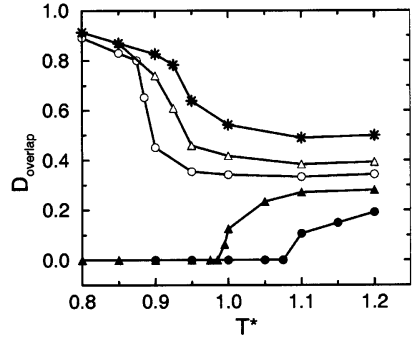

(b)
Figure 11. (a) Area per surfactant $A_{\mathrm{s}}$ and (b) extent of chain overlap

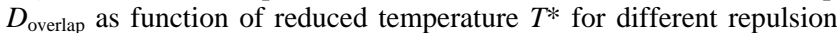
parameters $a_{\mathrm{hh}}$. The legend in graph (a) is valid for graph (b) as well.

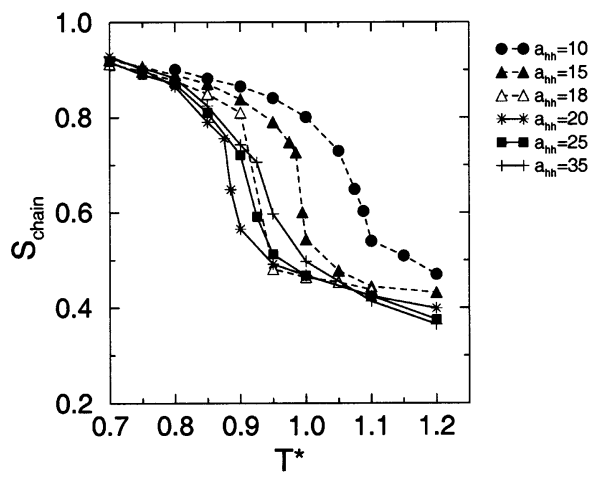

Figure 12. Chain order parameter $S_{\text {chain }}$ as function of reduced temperature $T^{*}$ for different values of the head-head repulsion parameter $a_{\mathrm{hh}}$. Dashed curves show a transition from the $L_{\beta}$ to the $L_{\alpha}$ phase, and solid curves show the transition from the $L_{\beta I}$ to the $L_{\alpha}$ phase.

while for bilayers in the $L_{\beta \text { I }}$ phase the area per molecule and chain overlap decrease.

In Figure 12, we plot $S_{\text {chain }}$ as a function of temperature for different values of the head-head repulsion parameter. Both in the $L_{\beta}$ and $L_{\beta 1}$ phases, the chains are highly ordered, whereas they get disordered with increasing temperature. The curves show that this transition from an ordered phase to a disordered one is very gradual. Much larger systems might be required to observe a sharp transition in these quasi-two-dimensional systems. This gradual transition makes it difficult to determine the exact location of the phase boundaries, and therefore, we used the inflection point as our definition of the phase boundary. The temperature at which the chains get disordered is the same as the temperature of the inflection point in $A_{\mathrm{s}}$ and $D_{\text {overlap. }}$. We define as the main transition temperature $T_{\mathrm{m}}$ the value of temperature at the inflection point of the shown curves. $T_{\mathrm{m}}$ is higher for bilayers in the $L_{\beta}$ phase than for bilayers in the $L_{\beta \mathrm{I}}$ phase. This is in agreement with experimental results. ${ }^{4}$

Besides investigating the effect of changing the head-head repulsion parameter, it is also interesting to vary the tail length of the surfactant. A similar analysis, as was presented for the surfactant $h t_{9}$, has been carried out for surfactant types $h t_{6}, h t_{7}$, and $h t_{8}$ (see Figure 13).

With increasing tail length, the transition temperatures $\left(L_{\beta 1}\right.$ $\rightarrow L_{\alpha}$ and $L_{\beta} \rightarrow L_{\alpha}$ ), both in the gel and in the interdigitated gel phase, increase: for longer surfactants, a higher temperature is needed to disorder the chains. This is consistent with experimental data on saturated phosphatidylcholines, ${ }^{2}$ which show an increase of the chain order/disorder transition temperature with increasing chain length.

Depending on the repulsion parameter, we obtain the two gel phases $L_{\beta 1}$ and $L_{\beta}$ for all tail lengths. For a high head-head repulsion parameter $\left(a_{\mathrm{hh}} \gg a_{\mathrm{hw}}\right)$, the system can gain energy by adding water particles between the heads. As a result, the

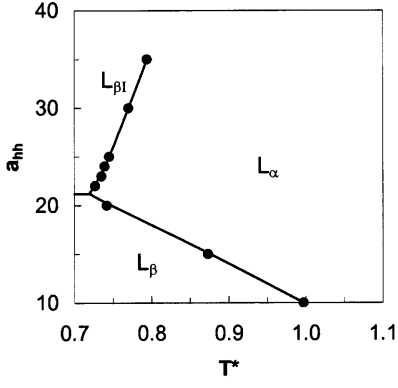

(a)

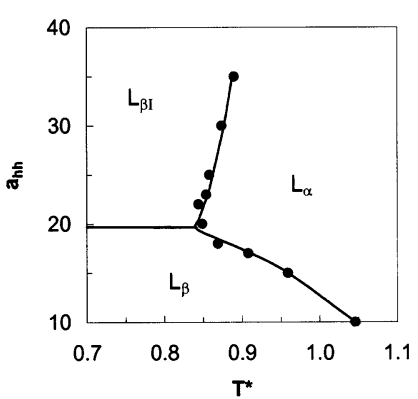

(c)

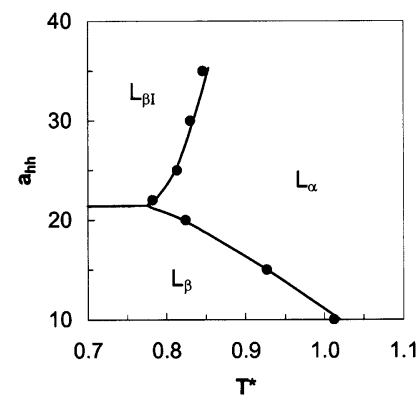

(b)

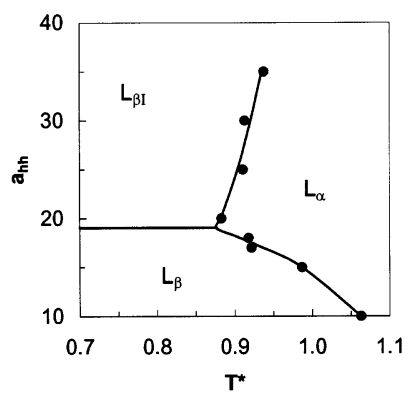

(d)
Figure 13. Phase diagrams for surfactants of different chain lengths: (a) $h t_{6}$, (b) $h t_{7}$, (c) $h t_{8}$, and (d) $h t_{9}$.

head-head distance increases, which stabilizes the interdigitated phase. At a low repulsion between the heads $\left(a_{\mathrm{hh}} \ll a_{\mathrm{hw}}\right)$, it is favorable to expel water from the headgroup region and the noninterdigitated $L_{\beta}$ phase is formed. Between we find $a_{\mathrm{hh}}^{*}$ for which the transition from $L_{\beta \mathrm{I}}$ to $L_{\beta}$ occurs. The difference between the two phases is that in the $L_{\beta \mathrm{I}}$ phase the tail ends are in direct contact with water, whereas in the $L_{\beta}$ phase the tail ends face each other. Therefore, the critical value of $a_{\mathrm{hh}}^{*}$ to induce interdigitation is higher than the value of $a_{\mathrm{hw}}$.

For surfactants $h t_{8}$ and $h t_{9}$, the $L_{\beta \mathrm{I}}$ phase occurs at slightly lower repulsion parameters than for surfactants $h t_{6}$ and $h t_{7}$. This is consistent with experimental results. ${ }^{9}$ They explain that, because the interdigitated phase is more closely packed than the noninterdigitated phase, the van der Waals energy is greater. This energy gain is proportional to the number of carbon atoms in the phospholipid chain, and thus, interdigitation becomes energetically more favorable for longer chains. Also in our simulations we observe that the interdigitated phase is more compact, and hence, $a_{\mathrm{hh}}^{*}$ decreases slightly with increasing tail length.

We observe hysteresis if we change $a_{\text {hh }}$ at a constant temperature: the bilayer can be both in the $L_{\beta}$ or in the $L_{\beta \mathrm{I}}$ phase, depending on the initial dimension of the area. The range of $a_{\mathrm{hh}}$ in which hysteresis occurs increases with decreasing temperature (see Figure 14). This suggests that the transition $L_{\beta}$ to $L_{\beta \mathrm{I}}$ is a first-order transition. In the phase diagrams of Figure 13, we define the phase found during decreasing temperature at a constant head-head repulsion parameter as the stable phase.

In Table 2, we compare the area per surfactant for different chain lengths and for two repulsion parameters: $a_{\mathrm{hh}}=15$ and $a_{\mathrm{hh}}=35$. Experimentally, it is found that, at the melting temperatures of various symmetric PCs, the area per lipid as a function of tail length is constant. ${ }^{32}$ At temperatures above the melting temperature, the area per lipid is found to decrease slightly in going from a tail length of 14 to a tail length of 18 carbons ${ }^{30}$ For both repulsion parameters, we observe that, in the liquid crystalline phase, the area per surfactant is constant 


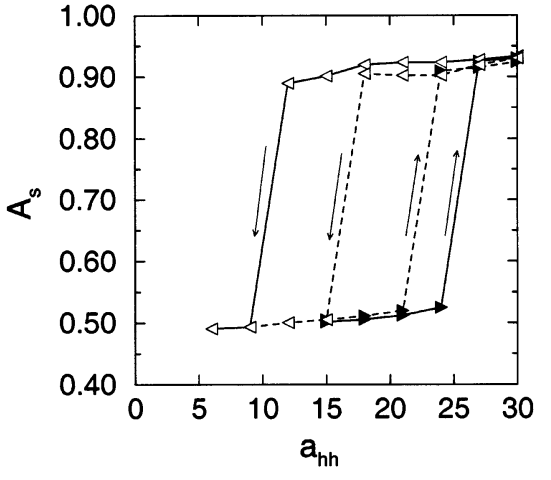

Figure 14. Hysteresis curves for the area per surfactant, $A_{\mathrm{s}}$ of the surfactant type $h t_{8}$, as function of the head-head repulsion parameter, $a_{\mathrm{hh}}$ at constant temperature $T^{*}=0.75$ (solid line) and $T^{*}=0.8$ (dashed line). Configurations of both phases $L_{\beta}$ (at $a_{\mathrm{hh}}=6$ ) and $L_{\beta \mathrm{I}}$ (at $a_{\mathrm{hh}}=$ 30) are taken as initial conditions, and $a_{\mathrm{hh}}$ is slowly increased or decreased, respectively.

TABLE 2: Area Per Surfactant as Function of Chain Length

\begin{tabular}{cccccc}
\hline & \multicolumn{2}{c}{$a_{\mathrm{hh}}=15$} & & \multicolumn{2}{c}{$a_{\mathrm{hh}}=35$} \\
\cline { 2 - 3 } \cline { 5 - 6 } surfactant type & $T^{*}=0.8$ & $T^{*}=1.0$ & & $T^{*}=0.8$ & $T^{*}=1.0$ \\
\hline$h t_{6}$ & 0.52 & 0.68 & & 0.87 & 0.83 \\
$h t_{7}$ & 0.51 & 0.68 & & 0.92 & 0.83 \\
$h t_{8}$ & 0.51 & 0.68 & & 0.93 & 0.84 \\
$h t_{9}$ & 0.51 & 0.68 & & 0.94 & 0.85
\end{tabular}

within the error $\left(\triangle A_{\mathrm{s}} / A_{\mathrm{s}}=5 \%\right)$, in agreement with the experimental results. The observed decrease in area per surfactant by Petrache et.al. is too small to allow for a detailed comparison with our mesoscopic model. This also holds in the gel phase for $a_{\mathrm{hh}}=15$. For $a_{\mathrm{hh}}=35$ in the interdigitated gel phase, we observe a slight increase of $A_{\mathrm{s}}$ with increasing chain length.

3.2. Double Tail Surfactants. In this section, we refine our model by introducing surfactants with a larger number of head beads and two hydrophobic tails (see Figure 2). We consider surfactants of various tail lengths ( $4-8$ hydrophobic beads) at different temperatures and at the same simulation conditions (200 surfactants, $\gamma=0$, and $a_{\mathrm{hh}}=35$ ) as used for the single tail surfactants. In these simulations we reproduce the low temperature $L_{\beta^{\prime}}$ phase and the high temperature $L_{\alpha}$ phase.

Interdigitation does not occur in bilayers of symmetrical chain phospholipids, but has to be induced. We also investigate whether, similar to experiments, we can induce interdigitation by adding a model ester-linkage to the surfactant headgroup. For this system we analyze different factors that might induce interdigitation.

3.2.1. Bilayer Structure. To study the temperature behavior, we cooled the system from $T^{*}=1.0$ to $T^{*}=0.1$ in steps of $\Delta T^{*}=0.05$. Figure 15 shows that the area per surfactant is increasing and the bilayer thickness is decreasing with increasing temperature. Similar results were obtained for other tail lengths and are in agreement with experimental results. ${ }^{30,32}$

At low temperatures, $T^{*}<0.3$, the $L_{\beta^{\prime}}$ phase is stable. The area per surfactant is equal for all tail lengths and the thickness increases linearly with tail length. The headgroup and the surrounding water are in a fluid phase, whereas the tails form a highly ordered bilayer consisting of two separated monolayers. In this temperature region, the chains are tilted at an angle $\theta$ with respect to the bilayer normal, to minimize contributions to the total energy from both headgroup and tail interactions. ${ }^{31,33,34}$ In our simulations, the tilt is about $25^{\circ}$ at $T^{*}=$ 0.2 , independent of tail length. Experimentally, a small increase

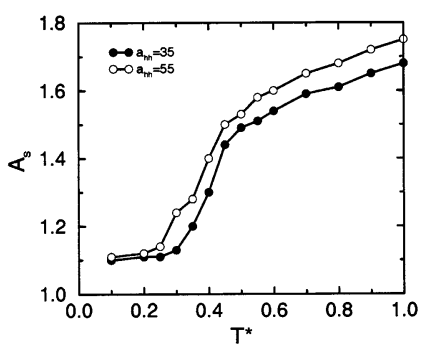

(a)

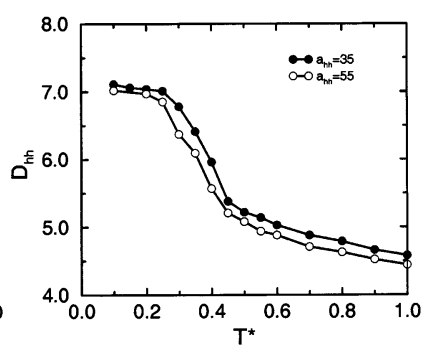

(b)
Figure 15. (a) Area per surfactant $A_{\mathrm{s}}$ and (b) bilayer thickness $D_{\text {hh }}$ as function of temperature $T^{*}$ for $h_{3}\left(t_{5}\right)_{2}$ with two different values of the headgroup repulsion parameter $a_{\mathrm{hh}}$.

of tilt angle with tail length was found. For instance TristramNagle et al. in ref 35 found that the tilt angle for PCs of different chain length increases from $\theta=32^{\circ}$ for 16 carbons to $\theta=35^{\circ}$ for 20 carbons in the chains.

Increasing the temperature above $T^{*}=0.3$ causes the melting of the bilayer from the $L_{\beta^{\prime}}$ to the $L_{\alpha}$ phase. The temperature at which the main transition to the $L_{\alpha}$ phase takes place increases with increasing tail length, as was also found experimentally. ${ }^{32,36}$ At temperatures higher than $T^{*}=0.6$, all of the bilayers are completely fluid, and above this temperature, the area per surfactant does not depend on tail length. According to Petrache et al., ${ }^{30}$ the main effect of increasing chain length is on the bilayer thickness, which is in agreement with our results. However, they find a sligthly decreasing area with increasing tail length. This decrease is too small to compare with our simulations, which show, within the accuracy of the data, a constant area per surfactant. They argue that the smaller area per surfactant is due to the larger interchain van der Waals attraction; that is, the longer chains keep the headgroups closer together. This is consistent with the fact that lipids with longer chains have higher melting temperatures. Indeed, we find higher melting temperatures with increasing tail length.

The behavior of the double tail surfactants deviates significantly from the behavior of the single tail surfactants. Although, for the head-head repulsion of 35 , the single tail surfactants show almost complete interdigitation at temperatures below $T^{*}$ $=1.0$, the double tail surfactants do not show interdigitation. Here we investigate whether an increase in headgroup separation is sufficient to induce interdigitation also for double tail surfactants.

In Figure 15, the area per surfactant and the thickness of the hydrophobic core are plotted as a function of temperature for surfactants of type $h_{3}\left(t_{5}\right)_{2}$ with two different repulsion parameters. For $a_{\mathrm{hh}}=55$, the area per surfactant is larger, whereas the thickness of the hydrophobic core is smaller at all temperatures compared to the same surfactant with $a_{\mathrm{hh}}=35$. An analysis of the order parameters shows that in the hightemperature region $\left(T^{*}>0.6\right)$ a larger disorder of the tails compensates for the extra space. Because of this increased disorder, a lower melting temperature $T_{\mathrm{m}}=0.35$ is found compared to $T_{\mathrm{m}}=0.4$ for $a_{\mathrm{hh}}=35$. In the low-temperature region $\left(T^{*}<0.3\right)$, the compensation for the extra space is obtained by a jump in the tilt angle from $\theta=25^{\circ}$ to $\theta=29^{\circ}$. However, despite the larger separation of the headgroups, no interdigitation was observed. Indeed, the tail overlap (eq 18) is zero for both parameters in the low-temperature region.

3.2.2. Induced Interdigitation. Phospholipids in a physiological environment are either zwitterionic or anionic at neutral $\mathrm{pH}$. However, cationic lipids play an important role in the delivery of DNA to eukaryotic cells. One way to obtain cationic lipids (for example 1,2-diacyl-P-O-ethyl-phosphatidylcholines) from 


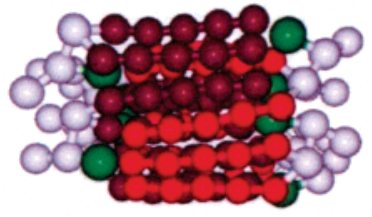

(a) (b)

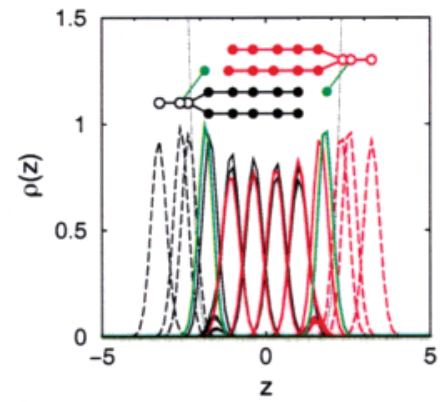

Figure 16. (a) Snapshot and (b) density profile of $t h_{3}\left(t_{5}\right)_{2}$ surfactants at $T^{*}=0.25$. The additional hydrophobic bead is green in both figures and the surfactant tails in one monolayer are darker red to distinguish them from the tails of the opposite monolayer. (See also the caption to Figure 6).

the zwitterionic PC lipids is to add an ethyl group at the $\mathrm{P}-\mathrm{O}$ in the headgroup region. These lipids show the spontaneous formation of a fully interdigitated bilayer in the low-temperature region. ${ }^{37,38}$ The interdigitation in these bilayers is induced by two effects: charge repulsion of the cationic headgroups and steric hindrance in the headgroup region caused by the presence of the ethyl group. Lewis et al. ${ }^{37}$ found that converting the zwitterionic headgroup into a positively charged headgroup by esterification is of main importance for inducing interdigitation and that the steric hindrance facilitates the interdigitation.

In our simulations, we investigated the influence of a charge in the headgroup and the presence of the ethyl group separately. In section 3.2.1, we have shown that repulsion between headgroups was not sufficient to induce interdigitation. To investigate the effect of adding an ethyl group to the surfactant headgroup, we attached an additional hydrophobic bead to the second bead $\left(h_{2}\right)$ of the headgroups of $h_{3}\left(t_{5}\right)_{2}$ and $h_{3}\left(t_{7}\right)_{2}$ to create the models $t h_{3}\left(t_{5}\right)_{2}$ and $t h_{3}\left(t_{7}\right)_{2}$ of the esterified phospholipid. In Figure 16, a snapshot of a patch of the bilayer and the corresponding density profile for $t_{3}\left(t_{5}\right)_{2}$ surfactants with $a_{\mathrm{hh}}=$ 55 at $T^{*}=0.25$ is shown. We observe that in this case the two monolayers are interdigitated.

The effect of the additional bead is 2-fold: it causes an increase in the distance between the headgroups and it changes the conformation of the headgroup. The additional hydrophobic bead sticks into the hydrophobic core, facing the tails of the surfactants in the opposite monolayer (see Figure 16), and forcing the second head bead to the interface. Because the additional head bead is located at the same depth in the bilayer as the first bead in the tail, given a tail length of 5 beads, interdigitation occurs up to four tail beads, as can be seen from the density profile (Figure 16b).

To analyze the effect of the repulsion parameter (which would mimic the charge on the headgroup or changing the salt concentration) and the tail length on the stability of this interdigitated gel, we compared the surfactant types $t h_{3}\left(t_{5}\right)_{2}$ and $t_{h}\left(t_{7}\right)_{2}$ with head-head repulsion parameters varying from $a_{\mathrm{hh}}$ $=5$ to $a_{\mathrm{hh}}=55$. In Figure 17, the corresponding phase diagrams are shown.

In the case of $t h_{3}\left(t_{5}\right)_{2}$ (Figure 17a), the bilayer is in the liquid crystalline phase at temperatures $T^{*}>0.5$. At low temperatures, different phases can be observed, as a function of $a_{\mathrm{hh}}$. For $a_{\mathrm{hh}}$ $\geq 30$, interdigitation is complete, whereas for all of the smaller repulsion parameters, coexistence between the $L_{\beta^{\prime}}$ and the $L_{\beta \mathrm{I}}$ phase was observed. At the lowest repulsion parameter $a_{\mathrm{hh}}=$ 5 , the surfactants demix; inverted micelles are formed within the hydrophobic core of the bilayer.

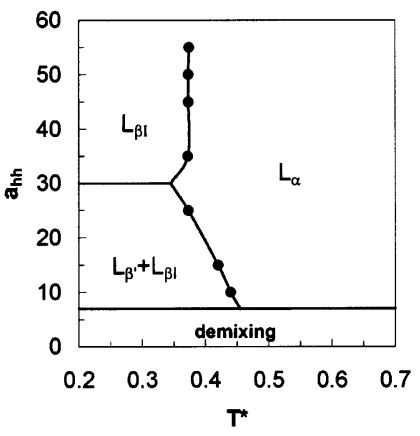

(a)

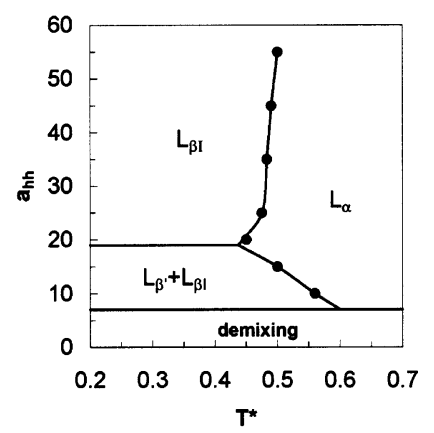

(b)
Figure 17. Phase diagrams for a bilayer consisting of (a) $t h_{3}\left(t_{5}\right)_{2}$ surfactants and (b) $t h_{3}\left(t_{7}\right)_{2}$ surfactants.

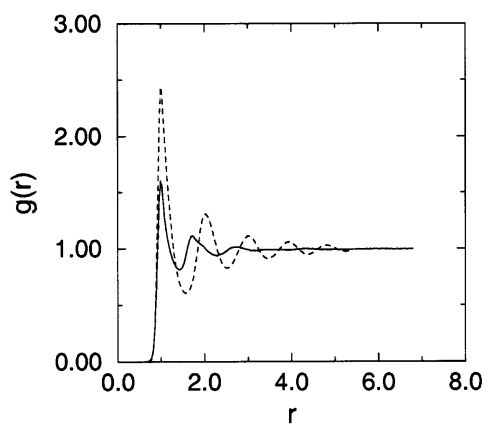

Figure 18. Two-dimensional radial distribution function of $t h_{3}\left(t_{5}\right)_{2}$ (solid line) and $h_{3}\left(t_{5}\right)_{2}$ (dashed line) surfactants at $T^{*}=0.25$.

Also in this system the transition temperature to the $L_{\alpha}$ phase increases with chain length. For the double tail surfactants in the interdigitated phase, both tail ends are in contact with the water phase, which needs to be compensated with a higher energy gain from the adsorption of water particles in the headgroup region; that is, $a_{\mathrm{hh}}^{*}$ is higher compared to single tail surfactants. The additional hydrophobic head bead, however, partially shields these tail ends, and therefore, the increase is not a factor of 2. Striking is that the tail length dependence is much more pronounced in these systems, which is a consequence of the tilted configuration these molecules have in the $L_{\beta^{\prime}}$ phase.

The explanation for the formation of the interdigitated phase can again be found in the larger area per surfactant. In Figure 18 , we compare for the surfactant $t h_{3}\left(t_{5}\right)_{2}$ the two-dimensional radial distribution function of the head-bead connecting the two tails $\left(h_{1}\right)$ for surfactants with and without the extra head bead (at $T^{*}=0.25$ and $a_{\mathrm{hh}}=55$ ). The bilayer of $h_{3}\left(t_{5}\right)_{2}$ surfactants is more "solidlike" than the bilayer of $t h_{3}\left(t_{5}\right)_{2}$ surfactants. Because of the interdigitation, in the second case, the headgroups have a larger surface area. Also the location of the peaks in both cases is different. For both surfactants the first peaks are located at the same distance, but the peak for $t h_{3}\left(t_{5}\right)_{2}$ is considerably lower than the peak of $h_{3}\left(t_{5}\right)_{2}$. The second peak for $t h_{3}\left(t_{5}\right)_{2}$ is shifted to the left, compared to $h_{3}\left(t_{5}\right)_{2}$. The additional hydrophobic bead is located between two headgroups which are at the distance of the second peak in $g(r)$. Thus, this second peak for $t h_{3}\left(t_{5}\right)_{2}$ indeed corresponds to nearest neighbors which are at a larger distance, because of the presence of the extra bead in the head, whereas for $h_{3}\left(t_{5}\right)_{2}$, it really corresponds to next-nearest-neighbors.

From our results, we can conclude that interdigitation induced by addition of an ester-linkage in the phospholipid headgroup region is mainly due to an increase of the headgroup surface area. This larger area is due to (1) steric hindrance of an extra 
group in the headgroup and (2) increase in headgroup repulsion. We have shown that the steric hindrance plays the crucial role in inducing interdigitation and is not just facilitating the interdigitation. ${ }^{37}$ The additional hydrophobic segment penetrates into the hydrophobic core, increasing the interface area, and it faces the tails of the opposite monolayer, screening them from the surrounding water. The increased repulsion between the headgroups alone is not sufficient to induce interdigitation but only facilitates the formation of a complete interdigitated phase.

\section{Conclusions}

In this work, we have shown that mesoscopic models of amphiphilic surfactants are able to describe the complex phase behavior of phospholipids bilayers. Because such models can be simulated very efficiently with dissipative particle dynamics, we could systematically study the bilayer properties as function of temperature and surfactant characteristics.

For both single tail and double tail model surfactants, we obtained different stable bilayer phases for a wide range of temperatures. We characterized the low-temperature phase as a gel phase, and for both models, we reproduced the main order/ disorder phase transition from gel to liquid crystalline. The zero surface tension simulation scheme is essential to observe these different phases.

We have shown that single tail surfactants spontaneously form a low temperature interdigitated phase for high enough values of the repulsion parameter between headgroups. A high value of headgroup repulsion parameter describes both the effect of the disproportion of polar and unpolar moieties in an amphiphilic surfactant and the increase in the electrostatic repulsion between surfactant headgroups due to the presence of salt. For the double tail surfactants, independent of the value of the headgroup repulsion parameter, the low temperature phase is a bilayer gel in which the hydrocarbon tails are tilted with respect to the bilayer normal, which is in agreement with what is experimentally found for phospholipid bilayers. We have shown that an interdigitated phase can be induced in a bilayer formed by double tail surfactants if the size of the headgroup is increased by adding an extra hydrophobic bead. From these results, we can conclude that even with a coarse grained model some level of chemical detail can be implemented and the effect of molecular structure on the properties of model bilayers can be studied. This makes coarse grained models a powerful and flexible tool to study a large variety of systems, like for example bilayers consisting of more than one surfactant molecular type.

Acknowledgment. These investigations are supported in part by The Netherlands Research Council for Chemical Sciences (CW), by the research program of the "Stichting voor Fundamenteel Onderzoek der Materie (FOM)", and by The Netherlands Organization for Scientific Research (NWO) through PIONIER.

\section{References and Notes}

(1) Katsaras, J.; Gutberlet, T. Lipid Bilayers. Structure and Interactions; Springer-Verlag: New York, 2001. 145.

(3) Slater, J. L.; Huang, C.-H. Prog. Lipid. Res. 1988, 27, 325-359.

(4) Furuike, S.; Levadny, V. G.; Li, S. J.; Yamazaki, M. Biophys. J. 1999, 77, 2015-2023.

(5) McIntosh, T. J.; McDaniel, R. V.; Simon, S. A. Biochim. Biophys. Acta 1983, 731, 109-114.

(6) McIntosh, T. J.; Lin, H.; Li, S.; Huang, C.-H. Biochim. Biophys. Acta 2001, 1510, 219-230.

(7) Adachi, T.; Takahashi, H.; Ohki, K.; Hatta, I. Biophys. J. 1995, $68,1850-1855$ 36

(8) Hata, T.; Matsuki, H.; Kaneshina, S. Biophys. Chem. 2000, 87, 25-

(9) Simon, S. A.; McIntosh, T. J. Biochim. Biophys. Acta 1984, 773, $169-172$.

(10) Marrink, S. J.; Mark, A. E. J. Phys. Chem. B 2001, 105, 61226127

(11) Goetz, R.; Lipowsky, R. J. Chem. Phys. 1998, 108, 7397-7409.

(12) Shelley, J. C.; Shelley, M. Y.; Reeder, R. C.; Bandyopadhyay, S.; Klein, M. L. J. Phys. Chem. B 2001, 105, 4464-4470.

(13) Shelley, J. C.; Shelley, M. Y.; Reeder, R. C.; Bandyopadhyay, S.; Moore, P. B.; Klein, M. L. J. Phys. Chem. B 2001, 105, 9785-9792.

(14) Venturoli, M.; Smit, B. Phys. Chem. Commun. 1999, 10

(15) Groot, R. D.; Rabone, K. L. Biophys. J. 2001, 81, 725-736.

(16) Shillcock, J. C.; Lipowsky, R. J. Chem. Phys. 2002, 117, 50485061.

(17) Hoogerbrugge, P. J.; Koelman, J. M. V. A. Europhys. Lett. 1992, 19, 155-160.

(18) Groot, R. D.; Warren, P. B. J. Chem. Phys. 1997, 107, 44234435 .

(19) Español, P.; Warren, P. Europhys. Lett. 1995, 30, 191-196.

(20) Jähnig, F. Biophys. J. 1996, 71, 1348-1349.

(21) Feller, S. E.; Pastor, R. W. Biophys. J. 1996, 71, 1350-1355.

(22) Feller, S. E.; Pastor, R. W. Biophys. J. 1999, 111, 1281-1287.

(23) Chiu, S. W.; Clark, M.; Balaji, V.; Subramaniam, S.; Scott, H. L.; Jakobsson, E. Biophys. J. 1995, 69, 1230-1245.

(24) Zhang, Y.; Feller, S. E.; Brooks, B. R.; Pastor, R. W. J. Chem. Phys. 1995, 103, 10252-10266.

(25) Rowlinson, J. S.; Widom, B. Molecular Theory of Capillarity; Clarendon Press: Oxford, 1982.

(26) Groot, R. D. Langmuir 2000, 16, 7493-7502.

(27) Flyvbjerg, H.; Petersen, H. G. J. Chem. Phys. 1989, 91, 461-466.

(28) Frenkel, D.; Smit, B. Understanding Molecular Simulations: from Algorithms to Applications; Academic Press: San Diego, CA, 2002.

(29) Cevc, G.; Marsh, D. Phospholipid bilayers: physical properties and models; John Wiley and Sons: New York, 1987.

(30) Petrache, H. I.; Dodd, S. W.; Brown, M. F. Biophys. J. 2000, 79, $3172-3192$

(31) Sun, W.-J.; Tristram-Nagle, S.; Suter, R. M.; Nagle, J. F. Biophys. J. 1996, 71, 885-891.

(32) Lewis, B. A.; Engelman, D. M. J. Mol. Biol. 1983, 166, 211-217.

(33) Nagle, J. F. J. Membr. Biol. 1976, 27, 233-250.

(34) McIntosh, T. J. Biophys. J. 1980, 29, 237-245.

(35) Tristram-Nagle, S.; Zhang, R.; Suter, R. M.; Worthington, C. R.; Sun, W. J.; Nagle, J. F. Biophys. J. 1993, 64, 1097-1109.

(36) Stümpel, J.; Eibl, H.; Niksch, A. Biochim. Biophys. Acta 1983, 727, $246-254$.

(37) Lewis, R. N. A. H.; Winter, I.; Kriechbaum, M.; Lohner, K.; McElhaney, R. N. Biophys. J. 2001, 80, 1329-3142.

(38) Winter, I.; Pabst, G.; Rappolt, M.; Lohner, K. Chem. Phys. Lipids 2001, 112, 137-150. 